\title{
Mitteilungen der ÖGGG
}

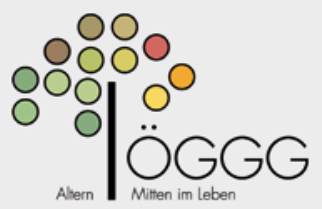

Z Gerontol Geriat 2012 · 45:684-684 DOI 10.1007/s00391-012-0412-5

(c) Springer-Verlag 2012

Österreichische Gesellschaft (ÖGGG)
Geschäftsstelle

Österreichische Gesellschaft

für Geriatrie und Gerontologie

Ilse Howanietz

Apollogasse 19

A-1070 Wien für Geriatrie und Gerontologie

Mitglied der International Association of Gerontology and Geriatrics (IAGG) ilse.howanietz@wienkav.at

www.geriatrie-online.at
Korrespondenzadresse

Prim. Dr. Katharina Pils

SMZ-Sophienspital

Apollogasse 19

A-1070 Wien

katharina.pils@wienkav.at

\section{Neue Mitglieder}

- Dr. Juliane Gruber

- OA Dr. Calin Gurguta

- Dr. Barbara Reidinger

\section{Professur für Geriatrie}

An der Medizinischen Universität Wien tritt mit 1. Oktober 2012 Prim. Univ. Prof. Dr. Marcus Köller die Professor für Geriatrie an. Eine ausführliche Vorstellung erfolgt in der nächsten Ausgabe.

\section{Publikationen}

Eva Mann, Alexander Vonbank, Heinz Drexel, Christoph H. Saely

Diabetes care among older adults in primary care in Austria.

A cross-sectional study

Swiss Med Wkly. 2012;142:w13646

Gosch M, Joosten-Gstrein B, Heppner HJ, Lechleitner M Hyponatremia in geriatric inhospital patients:

effects on results of a comprehensive geriatric assessment. Gerontology. 2012;58(5):430-40. Epub 2012 Jun 21.

Talasz H, Kremser C, Kofler M, Kalchschmid E, Lechleitner M, Rudisch A Proof of concept: differential effects of Valsalva and straining maneuvers on the pelvic floor.

Eur J Obstet Gynecol Reprod Biol. 2012 Jul 5.

Talasz H, Jansen SC, Kofler M, Lechleitner M

High prevalence of pelvic floor muscle dysfunction in hospitalized elderly women with urinary incontinence.

Int Urogynecol J. 2012 Sep;23(9):1231-7. Epub 2012 Jan 4.

\section{Veranstaltungen und Kongresse}

15. November 2012, 19.00 Uhr s.t. Wissenschaftlichen Sitzung der ÖGGG und des LBI für Altersforschung

„Ludwig Boltzmann-Institut für Altersforschung 1972-2012“

Gesellschaft der Ärzte, Frankgasse 8, 1090 Wien

17. November 2012

Medicine of Ageing 2012

Stadtforum BTV, Gilmstrasse, Innsbruck

http://www.gerimed.at

21.-23. März 2013

Wiener Geriatriekongress „Alter(n) - Chance und Herausforderung” Congress Center, Messe Wien

http://www.geriatriekongress.at 